\title{
Uma Visão do Ensino de Computação nos Cursos Técnicos Integrados ao Ensino Médio em campi do Instituto Federal do Rio de Janeiro: Uso de Programação no Apoio ao Aprendizado
}

\author{
Marcelo Simas Mattos ${ }^{1}$, Sergio Crespo Coelho da Silva Pinto ${ }^{1,2}$ \\ ${ }^{1}$ Programa de Pós-Graduação em Engenharia de Produção e Sistemas Computacionais - \\ Universidade Federal Fluminense (UFF), Campus de Rio das Ostras - Rua Recife, s/n, \\ Jd. Bela Vista - CEP 28895-532 - Rio das Ostras, RJ - Brasil \\ ${ }^{2}$ Departamento de Computação - Instituto de Ciência e Tecnologia Universidade \\ Federal Fluminense (UFF), Campus de Rio das Ostras - Rua Recife, s/n, Jd. Bela Vista \\ - CEP 28895-532 - Rio das Ostras, RJ - Brasil \\ marcelo.mattos@ifrj.edu.br, screspoeid.uff.br
}

\begin{abstract}
This article describes an investigation on the use of computer programming to support the learning of disciplines of the common core of high school in two campuses from the Federal Institute of Rio de Janeiro (IFRJ). It was made a literature review in Brazilian Journal of Informatics in Education (RBIE), in New Technologies in Education (RENOTE) and at the Workshop of Informatics in School (WIE), about the programming resources used in school education and in graduations. With the application of a questionnaire to teachers of campuses chosen, it sought to verify the development projects and understanding of teachers about the use of computer programming in school teaching and learning.
\end{abstract}

Resumo. Este artigo descreve uma investigação sobre o uso de programação de computadores como apoio ao aprendizado de disciplinas do núcleo comum do Ensino Médio em dois "campi" do Instituto Federal do Rio de Janeiro (IFRJ). Foi feito um levantamento bibiográfico na Revista Brasileira de Informática na Educação (RBIE), na Revista Novas Tecnologias na Educação (RENOTE) e no Workshop de Informática na Escola (WIE), sobre os recursos de programação usados na educação escolar e nas graduações. Com a aplicação de um questionário aos professores dos "campi" escolhidos, buscou-se verificar os projetos que vêm sendo desenvolvidos e os entendimentos dos docentes sobre o uso de programação de computadores no ensino-aprendizagem escolar.

\section{Introdução}

O ensino de programação nas escolas de Ensino Fundamental e Ensino Médio se apresenta como uma alternativa para motivar e engajar o aluno no processo de ensinoaprendizagem, tornando-o mais criativo e estimulante (Scaico et. al. 2013, Silva et. al. 2015). No entanto, em um sentido mais amplo, há que se considerar o que Wing (2006) descreve como pensamento computacional. Segundo este autor, esse tipo de pensamento 
é necessário para a aprendizagem de programação, e pode se apresentar como uma alternativa para desenvolver habilidades de leitura, escrita e aritmética em jovens. Ele ainda descreve em linhas gerais que o pensamento computacional envolve resolver problemas, conceber sistemas, e compreender do comportamento humano, que se desenham como conceitos fundamentais para a Ciência da Computação.

Trabalhos recentes em âmbito nacional vêm propondo a introdução de conceitos e práticas próprias da Ciência da Computação em disciplinas escolares regulares da educação básica (Ferreira et. al. 2015, França e Amaral 2013, Hinterholz e Cruz 2015), incentivando os alunos a desenvolverem o pensamento computacional. Desses estudos, em se tratando especificamente do ensino de programação na educação escolar há indicação de níveis mais altos de motivação e empenho dos estudantes com essas experiências em suas aprendizagens, mas na revisão sistemática realizada por Silva et. al. (2015) é alertado que para que se tenha uma aplicação efetiva e em escala no ensino básico ainda se requer mais pesquisa e o desenvolvimento de novas abordagens.

Os conceitos de Ciência da Computação vêm influenciando outras áreas, como por exemplo: aprendizagem de máquina na Estatística; conceitos de Ciência da Computação na Biologia; nanocomputing na Química e computação quântica na Física (Wing 2006). Entretanto, a Computação, geralmente, não é integrada aos currículos formais escolares (Rodrigues et. al. 2015, Hinterholz e Cruz 2015).

Dois campi do IFRJ foram escolhidos para serem investigados, o Campus Arraial do Cabo e o Campus Nilópolis. Buscou-se verificar o uso de recursos de programação de computadores em seus cursos técnicos integrados ao Ensino Médio, a partir das atuações dos docentes. Foi pedido que professores que lecionam nesses cursos, de ambos os campi, respondessem a um questionário sobre o uso de programação como apoio ao aprendizado, com o intuito de averiguar como estão sendo apropriados os conceitos de Computação, especificamente como a programação está inserida no contexto curricular do IFRJ.

\section{Contextualização da Instituição investigada}

O Instituto Federal de Educação, Ciência e Tecnologia do Rio de Janeiro (IFRJ), foi criado a partir da publicação da Lei n ${ }^{0} 11.892$ de dezembro de 2008 mediante a transformação do Centro Federal de Educação Tecnológica de Química de Nilópolis. Entre as características descritas na lei de criação podemos destacar três que justificam este estudo, que são: "desenvolver a educação profissional e tecnológica como processo educativo e investigativo de geração e adaptação de soluções técnicas e tecnológicas às demandas sociais e peculiaridades regionais"; "constituir-se em centro de excelência na oferta do ensino de ciências, em geral, e de ciências aplicadas, em particular, estimulando o desenvolvimento de espírito crítico, voltado à investigação empírica" e "realizar e estimular a pesquisa aplicada, a produção cultural, o empreendedorismo, o cooperativismo e o desenvolvimento científico e tecnológico;" (Brasil, 2008).

Seguindo os preceitos das três características destacadas da lei de criação dos Institutos Federais (IFs) e o objetivo geral deste artigo, procurou-se verificar quais soluções técnicas e tecnológicas já foram utilizados, o que se realiza no momento e quais são as expectativas futuras sobre o uso de programação como apoio ao 
aprendizado nas diversas disciplinas dos cursos técnicos integrados ao Ensino Médio do IFRJ.

O IFRJ oferece cursos técnicos de nível médio, graduação, especialização e mestrado. Entre os níveis oferecidos, escolhendo-se o nível médio nos dois campi, se têm os seguintes cursos técnicos integrados ao Ensino Médio: no Campus Arraial do Cabo, o Curso Técnico em Montagem e Suporte em Informática (Proeja) e o Curso Técnico em Informática; no Campus Nilópolis, o Curso Técnico em Montagem e Suporte em Informática (Proeja), o Curso Técnico em Controle Ambiental e o Curso Técnico em Química.

\section{Procedimentos Adotados no Estudo}

No presente trabalho foi adotada a metodologia de estudo de caso, consistindo em um problema a ser investigado profundamente em um determinado tempo a partir das etapas: delimitação da unidade-caso, coleta de dados, seleção, análise e interpretação dos dados e elaboração do relatório do caso (Ventura 2007).

$\mathrm{Na}$ delimitação da pesquisa foi definido que seria investigado o uso de programação de computadores como apoio ao aprendizado dos estudantes em disciplinas propedêuticas (Disciplinas do Núcleo comum do Ensino Médio) a partir das atuações dos docentes dos cursos técnicos integrados ao Ensino Médio nas unidades Campus Arraial do Cabo e Campus Nilópolis do IFRJ.

Inicialmente foi realizada uma pesquisa bibliográfica nas fontes RENOTE, RBIE e WIE buscando estudos que tenham investigado o uso de recursos de programação de computadores como apoio ao aprendizado. Na sequência foi proposto aos professores dos cursos técnicos integrados ao Ensino Médio do IFRJ que respondessem um questionário sobre suas experiências com recursos de programação. Esse questionário permaneceu à disposição para resposta voluntária durante 16 dias.

O questionário aplicado foi de caráter qualitativo com perguntas de respostas fechadas, semifechadas e abertas, que serviram para investigar se os professores conheciam os recursos propostos nas alternativas, além de pedir que relatassem suas experiências, ações, projetos e impressões sobre o tema programação como apoio ao aprendizado em disciplinas do Ensino Médio.

\subsection{Pesquisa Bibliográfica}

Para realizar a investigação sobre o uso de programação como apoio ao aprendizado, o presente estudo se balizou por publicações científicas nacionais. Foram buscadas publicações dos últimos cinco anos (2011 a 2015) na Revista Brasileira de Informática na Educação (RBIE) e a Revista Novas Tecnologias na Educação (RENOTE) e nos três últimos anos no Workshop de Informática na Escola (WIE).

No levantamento bibliográfico foram selecionados estudos que propunham ensinar programação como recurso para o aprendizado de conceitos de disciplinas escolares ou para o aprendizado de programação no ensino superior. Com esse levantamento esperava-se conhecer as iniciativas que vem sendo adotadas como alternativas às formas tradicionalmente usadas para o ensino de programação. 
A leitura e extração de informações dos artigos selecionados serviram para elaborar as perguntas do questionário aplicado aos professores do IFRJ, com a devida coerência com que vem sendo pesquisado em estudos ligados ao pensamento computacional. Nas fontes RENOTE e RBIE foi usado o mesmo termo de busca: (educação OR ensino) AND ("programação de computadores" OR "linguagem de programação" OR programação). Para escopo de busca escolheu-se "todos", e se configurou para buscar artigos dos últimos cinco anos (2011 a 2015). A ferramenta de busca da RENOTE retornou 21 artigos e a RBIE 16 artigos, fazendo a leitura do título e abstract, nos resultados destas duas revistas, foram selecionados respectivamente $18 \mathrm{e}$ 14 artigos. Seguiu-se para a fase de inclusão ou exclusão pelo critério já relatado, foi excluído um artigo da RENOTE e dois artigos na RBIE. Sendo assim, essas duas fontes juntas totalizaram 29 artigos selecionados. Na fonte WIE foram selecionados artigos de 2013 a 2015. Primeiramente realizou-se a seleção através da leitura de títulos e abstract, na sequência foi usado o mesmo critério de inclusão e exclusão adotado nas revistas. Com isso, foi extraído 19 artigos na WIE.

A extração de informações foi voltada para se descobrir quais os recursos de programação foram citados e onde se aplicaram, resultando nas seguintes perguntas:

Questão 1 - Quais recursos de programação foram citados nos textos pesquisados?

Questão 2 - Quantos artigos investigaram a aplicação em cursos, oficinas ou projetos para cada recurso extraídos da questão 1? E quais foram os recursos usados nesses trabalhos?

Questão 3 - Em quais disciplinas do currículo escolar os recursos de programação foram aplicados?

\subsection{Resultados da Pesquisa Bibliográfica}

Alguns recursos e iniciativas citados foram: o Code.org, CodeHS, Codecademy, CoderDojo, Girls Who Code, Black Girls Code, Computer Science on Air, PyGame, RoboMind, Lego Mindstorms, Takkou, Scratch, Alice, GameMaker, Construct 2 e Flash. Realizando a contagem de cada artigo onde se mencionou os recursos se obteve os números ilustrados na Figura 1.

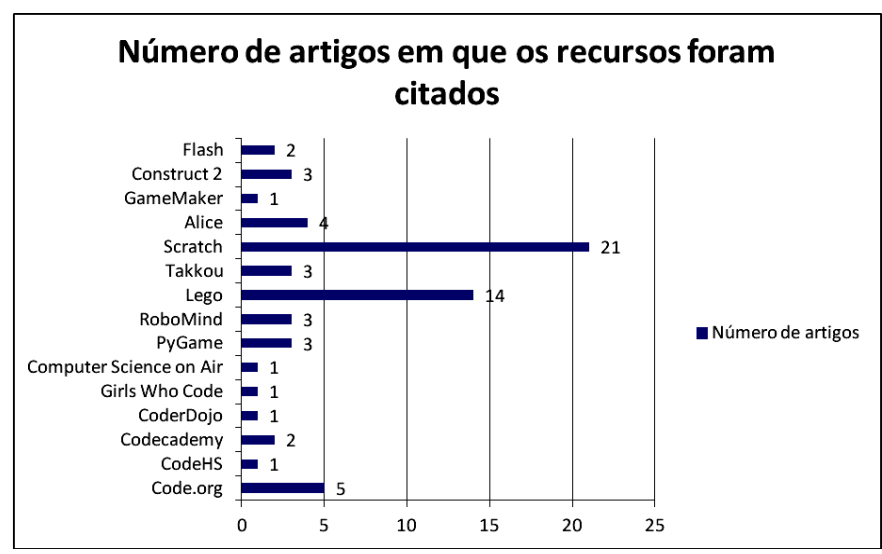

Figura 1. Número de artigos que fazem menção a cada um dos recursos.

Considerando os 48 artigos analisados, verificou-se que a maioria faz referência ao Scratch, identificado em 21 (43,8\%), na sequência Lego em 14 (29,2\%) e Code.org 
V Congresso Brasileiro de Informática na Educação (CBIE 2016)

Anais do XXII Workshop de Informática na Escola (WIE 2016)

em 5 (10,4\%). Em Lessa et. al. (2015) se tem resultados semelhantes, contudo naquele artigo foi pesquisado apenas na fonte WIE e se buscou quais ferramentas estavam sendo usadas em "Programação de Computadores" e em "Robótica Educativa" na escola.

Respondendo à Questão 2, temos que a maioria dos artigos que investigaram a aplicação dos recursos de programação fizeram uso do Scratch, foram 15 artigos (31,3\% dos artigos analisados), seguido do Lego Mindstorms e GameMaker com dois artigos cada (4,2\% dos artigos analisados). Pode-se identificar na Figura 2 que entre ambientes de programação ligados Robótica Educacional os números estão diluídos entre a ferramenta comercial Lego Mindstorms, e as não comerciais: Mblock, Squeak Etoys e DuinoBlocks (Alves, Sampaio e Elia 2015).

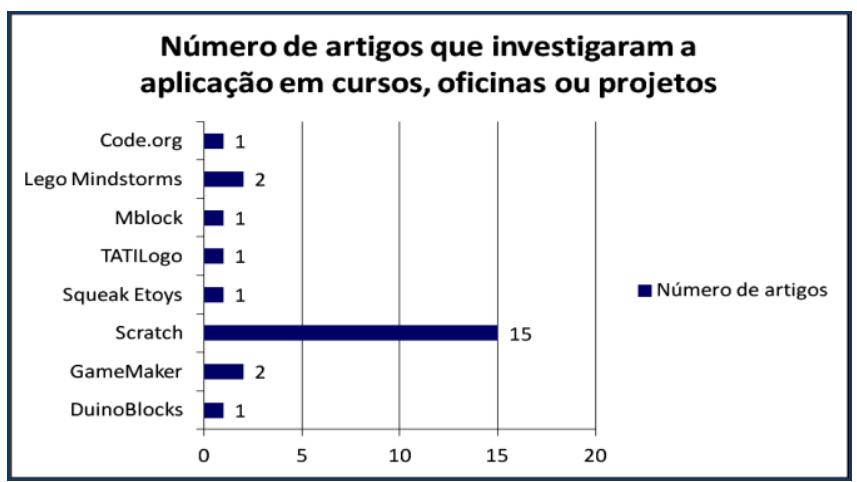

Figura 2. Artigos que investigaram a aplicação dos recursos de programação.

Os resultados representados pela Figura 1 e Figura 2 auxiliaram na escolha das alternativas do questionário, no que se refere ao que atualmente as pesquisas vêm estudando sobre o uso de programação como apoio ao aprendizado.

Os artigos em que os autores definiram claramente a disciplina escolar em que estava inserido o recurso, teve como resultado o ilustrado no gráfico da Figura 3. Podese constatar que a maioria foi aplicada em Matemática (6 artigos), seguidos de Artes e Língua Portuguesa e Literatura (2 artigos cada) e Inglês, Biologia, Química, Física e Educação Física com 1 artigo cada. As disciplinas Sociologia, Geografia, Filosofia e História não foram identificadas nos artigos.

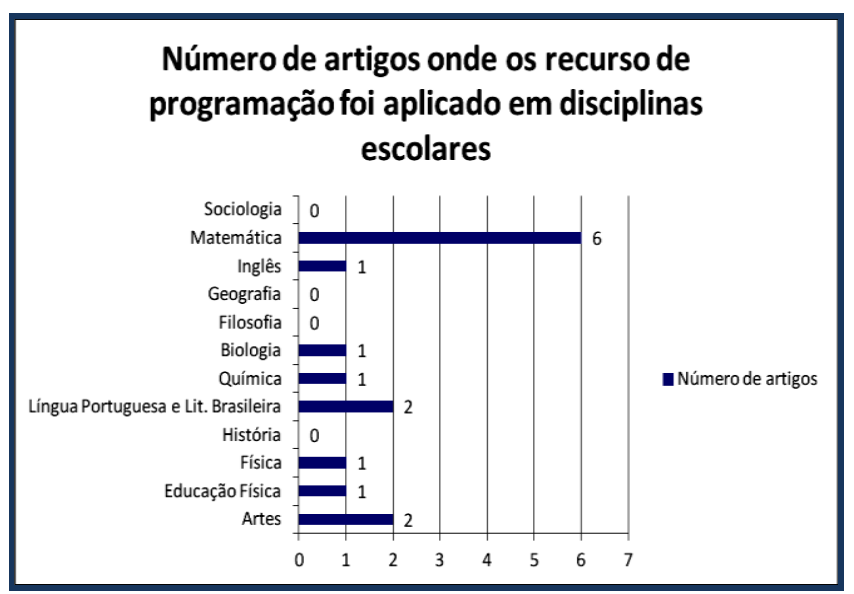

Figura 3. Aplicação dos recursos em disciplinas escolares. 
V Congresso Brasileiro de Informática na Educação (CBIE 2016)

Anais do XXII Workshop de Informática na Escola (WIE 2016)

O resultado da Figura 3 indica que houve pesquisas em várias disciplinas escolares nos últimos anos, porém em pequena quantidade em algumas áreas ou inexistente em outras. Estes resultados reforçam a necessidade de que mais pesquisas sejam realizadas nessa área.

\subsection{Questionário}

Através da ferramenta "Formulários Google" foi elaborado um questionário para ser enviado aos professores dos dois campi do IFRJ. Na ferramenta se configurou para que os pesquisados não fossem identificados e a adesão a ele era de livre escolha dos respondentes. O formulário teve as perguntas que estão na tabela $1 \mathrm{com}$ as respectivas configurações para "Tipo de alternativa", "Alternativas" e "Campo de descrição", mostrados nesta tabela (onde se vê um traço, não se aplica o campo).

Tabela 1. Perguntas que foram inseridas na ferramenta de formulários da Google

\begin{tabular}{|c|c|c|c|}
\hline Pergunta & $\begin{array}{c}\text { Tipo de } \\
\text { alternativa }\end{array}$ & Alternativas & $\begin{array}{l}\text { Campo de } \\
\text { descrição }\end{array}$ \\
\hline 1. Qual é o seu campus? & $\begin{array}{c}\text { Múltipla } \\
\text { escolha }\end{array}$ & $\begin{array}{l}\text { Campus Arraial do Cabo; Campus } \\
\text { Nilópolis. }\end{array}$ & 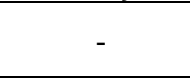 \\
\hline $\begin{array}{l}\text { 2. Qual(is) disciplina(s) você leciona no } \\
\text { IFRJ? }\end{array}$ & $\begin{array}{l}\text { Caixa de } \\
\text { seleção }\end{array}$ & $\begin{array}{l}\text { Artes; Biologia; Educação Física; } \\
\text { Filosofia; Física; Geografia; } \\
\text { História; Inglês; Língua } \\
\text { Portuguesa e Lit. Brasileira; } \\
\text { Matemática; Química; Sociologia; } \\
\text { Disciplina(s) técnica(s); Outro } \\
\text { (Especifique). }\end{array}$ & $\begin{array}{l}\text { Se você } \\
\text { marcou } \\
\text { "Disciplina(s) } \\
\text { técnica(s)", } \\
\text { especifique: }\end{array}$ \\
\hline $\begin{array}{l}\text { 3. Quais linguagens de programação você já } \\
\text { usou pelo menos uma vez? }\end{array}$ & $\begin{array}{l}\text { Caixa de } \\
\text { seleção }\end{array}$ & $\begin{array}{l}\text { Java; C/C++; C\#; Javascript; PHP; } \\
\text { Python; Pascal; Visual Basic; } \\
\text { Fortran; Matlab; Cobol; Perl; } \\
\text { Ruby; Outro (Especifique). }\end{array}$ & - \\
\hline $\begin{array}{l}\text { 4. Pesquisas na área de ensino de } \\
\text { programação voltadas para a educação } \\
\text { básica e superior vêm propondo o uso de } \\
\text { recursos como os listados a seguir, marque } \\
\text { os que você conhece. }\end{array}$ & $\begin{array}{l}\text { Caixa de } \\
\text { seleção }\end{array}$ & $\begin{array}{l}\text { MIT Scratch; Flash; Lego } \\
\text { Mindstorms; Visualg; PyGame; } \\
\text { Alice; Squeak Etoys; Construct 2; } \\
\text { Recursos na Web (como o } \\
\text { Codeacademy, CoderDojo, Girls } \\
\text { who code, Code.org, Computer } \\
\text { Science for High School); MIT } \\
\text { App Inventor; Outro (Especifique). }\end{array}$ & - \\
\hline $\begin{array}{l}\text { 5. Você já usou algum recurso de } \\
\text { programação em disciplina que leciona no } \\
\text { IFRJ? }\end{array}$ & $\begin{array}{l}\text { Múltipla } \\
\text { escolha }\end{array}$ & Sim; Não. & $\begin{array}{l}\text { Caso tenha } \\
\text { marcado sim, } \\
\text { especifique o } \\
\text { recurso: }\end{array}$ \\
\hline $\begin{array}{l}\text { 6. Você já realizou ou está realizando algum } \\
\text { projeto que faz uso de programação como } \\
\text { apoio ao aprendizado em disciplinas } \\
\text { propedêuticas? }\end{array}$ & $\begin{array}{l}\text { Múltipla } \\
\text { escolha }\end{array}$ & Sim; Não. & $\begin{array}{l}\text { Caso tenha } \\
\text { marcado sim, } \\
\text { favor } \\
\text { descrever } \\
\text { brevemente } \\
\text { o(s) } \\
\text { projeto(s). }\end{array}$ \\
\hline $\begin{array}{l}\text { 7. Quais benefícios que você acredita que a } \\
\text { tarefa de programar pode oferecer para } \\
\text { melhorar o aprendizado em outras áreas que } \\
\text { não sejam específicas de programação? }\end{array}$ & $\begin{array}{l}\text { Texto de } \\
\text { resposta } \\
\text { longa }\end{array}$ & - & - \\
\hline $\begin{array}{l}\text { 8. Em sua opinião, o desenvolvimento de } \\
\text { alguma atividade/projeto com uso de } \\
\text { programação de modo interdisciplinar na } \\
\text { disciplina que você leciona no IFRJ poderia } \\
\text { melhorar o aprendizado dos conteúdos? }\end{array}$ & $\begin{array}{l}\text { Múltipla } \\
\text { escolha }\end{array}$ & Sim; Não & $\begin{array}{l}\text { Justifique a } \\
\text { sua resposta: }\end{array}$ \\
\hline
\end{tabular}


V Congresso Brasileiro de Informática na Educação (CBIE 2016)

Anais do XXII Workshop de Informática na Escola (WIE 2016)

\section{Análise das respostas ao questionário}

O questionário foi enviado para o e-mail institucional de 40 docentes do Campus Arraial do Cabo e para uma lista de e-mail de docentes do Campus Nilópolis que continha 165 endereços, totalizando 205 professores.

O número de docentes que responderam ao questionário foram 42 pesquisados, 28 (66,7\%) pesquisados do Campus Arraial do Cabo e 14 de Nilópolis (33,3\%).

Das 13 possibilidades de áreas para serem indicadas pelos docentes, 12 teve representantes, apenas a disciplina de Artes não houve resposta de professores. A distribuição de respostas por áreas foram as seguintes: $12(28,5 \%)$ marcaram a alternativa área técnica (10 eram de disciplinas de Informática, 1 de Empreendedorismo e 1 de Meio Ambiente); 6 (14,3\%) de Química; 5 (11,9\%) de Geografia; 3 (7,1\%) para cada uma das disciplinas Biologia, Física e Matemática; 2 (4,8\%) para cada uma das disciplinas História, Inglês, Língua Portuguesa e Sociologia; e $1(2,4 \%)$ para cada uma das disciplinas Educação Física e Filosofia.

O questionamento das linguagens de programação que os pesquisados usaram pelo menos uma vez foi respondido por 17 professores. As linguagens de programação mais lembradas pelos pesquisados foram as que tradicionalmente são usadas nas graduações para o ensino de programação, como $\mathrm{C} / \mathrm{C}++(13 ; 76,5 \%)$, Pascal (13; $76,5 \%)$ e Java (12; 70,6\%). Na sequência vieram: Visual Basic indicado por $11(64,7 \%)$; Javascript e Python indicados por $10(58,8 \%)$ pesquisados cada; PHP e Matlab por 9 (52,9\%); C\# por 6 (35,3\%); Fortran, Cobol e Ruby por $3(17,6 \%)$; e por último Perl indicado por $1(5,9 \%)$ pesquisado. Para o campo "Outro" ainda foram citados pelos professores algumas linguagens, como se segue: o pesquisado de numeral 18 indicou o ActionScript; o pesquisado 30 indicou Clipper e o Delphi (Object Pascal); o pesquisado 37 indicou o Gw Basic e Scilab; e o pesquisado 40 a linguagem HTML.

Foram oferecidas aos docentes alternativas de recursos de programação que estão sendo propostos para a educação básica e superior, pedindo que os professores marcassem os que conheciam. Apenas 17 dos pesquisados responderam essa questão, 15 $(88,2 \%)$ conheciam o Flash, 9 (52,9\%) recursos na Web, 7 (41,2\%) o MIT Scratch e o Visualg, $4(23,5 \%)$ o Lego Mindstorm, 3 (17,6\%) o Pygame, 2 (11,8\%) o MIT App Inventor e $1(5,9 \%)$ para cada um dos recursos Alice, Squeak Etoys e Construct 2. Ainda foram especificados para a alternativa "Outro", os recursos Blender, Code School, Cousera, Nanodegree, Udemy e Logo. É importante destacar que, dos 17 pesquisados que conheciam pelo menos um recurso, $8(47,1 \%)$ eram professores da área de Informática e $9(52,9 \%)$ eram de disciplinas propedêuticas. Todavia, $5(29,4 \%)$ dos professores de área propedêuticas conheciam apenas o Flash, estes professores eram das disciplinas Física, Língua Portuguesa, Matemática e Química. Para os outros 4 professores segue o nome da disciplina e indicação do que conhecia: Química - Flash e Recursos na Web; Física - Flash, Lego Mindstorms; Inglês - Recursos na Web e Educação Física - Flash e Recursos na Web. Vale alertar que o Flash é um software proprietário, sendo assim, para os docentes realizarem iniciativas com o uso dele seria necessário adquirir licenças.

Questionou-se aos professores se eles já haviam usado recursos de programação em disciplina que leciona no IFRJ, 29 (69\%) professores disseram não e 13 (31\%) sim. 
Dos treze pesquisados que haviam usado, 8 eram docentes de disciplinas de informática, os outros eram: dois de Física, um de Química, um de Matemática e um de Educação Física. Quando questionados se já realizou ou está realizando projeto que faz uso de programação como apoio ao aprendizado de disciplinas do núcleo comum do Ensino Médio (disciplinas propedêuticas), $35(83,3 \%)$ pesquisados disseram que não e 7 $(16,7 \%)$ disseram sim, seis deles informaram os seguintes projetos: "Jogo de computador educativo"; "Proposta no ensino de Química Orgânica com o uso de algumas especiarias como tema transversal"; "Sites e animações em Flash, e vídeos 3D (Blender) para o ensino de disciplinas propedêuticas"; "Iniciando um projeto em conjunto com a professora de Inglês usando ambientes de codificação on-line"; "Projeto de automação de uma biblioteca", "No ensino de Física usei o Matlab para resolver questões". Para a presente pesquisa, esse resultado foi bom, pois se tem precedentes na instituição, o que contribui para possíveis futuras propostas.

Os professores responderam sobre quais os benefícios que eles acreditam que a tarefa de programar poderia oferecer para melhorar o aprendizado em outras áreas que não fossem específicas de programação. Com as frases dos 26 (61,9\%) docentes que responderam sobre os benefícios, foi gerada uma nuvem de palavras ilustrada pela Figura 4. As palavras que estão em evidência como raciocínio, lógico, conhecimento, desenvolvimento, capacidade e desenvolvimento, se estruturadas em uma frase representam as principais ideias sobre o pensamento computacional. Em segundo nível de frequência estão palavras como abstração, síntese e lógica que também estão presentes em definições sobre o pensamento computacional. Este resultado dá indícios de que, de uma forma geral, os docentes conheciam os presumidos benefícios.

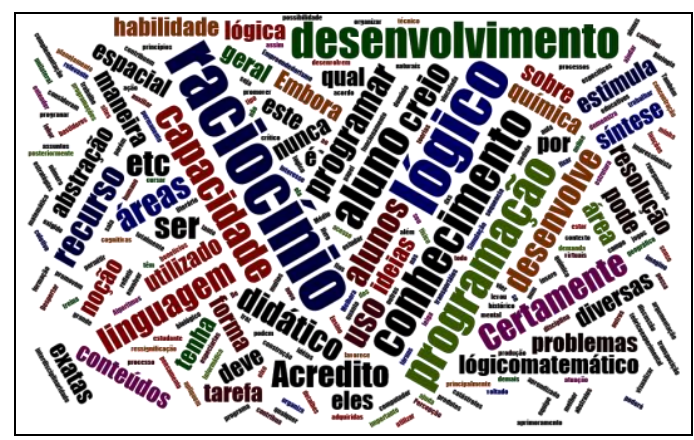

Figura 4. Benefícios que a tarefa de programar pode oferecer.

Quando os pesquisados foram perguntados se achavam que o desenvolvimento de alguma atividade/projeto com o uso de programação de modo interdisciplinar na disciplina que leciona no IFRJ poderia melhorar o aprendizado. A maioria disse sim (40; $95,2 \%)$, que poderia melhorar e $2(4,8 \%)$ não. Foi pedido para os pesquisados justificarem suas respostas, com base nelas foi gerada a nuvem de palavras representada na Figura 5. As principais ideias podem ser captadas por palavras com mais ênfase, como acredito, programação, área, trabalho, conteúdos, pode, estudantes, ciências, melhor, ambiente e disciplina. A análise das justificativas desta questão em conjunto com o resultado da pergunta fechada, faz presumir que os docentes, em sua maioria, acreditam que os recursos se oferecem como uma alternativa para o aprendizado nas disciplinas que lecionam. 


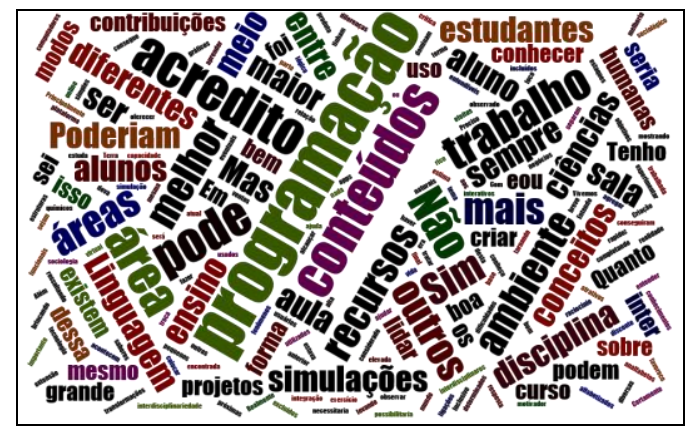

Figura 5. Justificativa sobre projetos interdisciplinares

Os resultados mostrados nas figuras que representam as últimas questões apontam justificativas positivas sobre o uso de programação como apoio ao aprendizado, contudo alguns outros aspectos foram verificados, como os 10 (24\%) professores que optaram por não responder a questão 7, possivelmente mostrando que desconheciam as possibilidades educacionais da programação em suas disciplinas e outros $6(14,3 \%)$ deixando claro que desconheciam os possíveis benefícios, com as seguintes respostas: "Não tenho ideia", "Não posso opinar"; "Não faço ideia", "não possuo conhecimentos para aplicar esse uso" e "Não sei responder". Na questão 8, foram $6(14,3 \%)$ os pesquisados que optaram por não responder e $1(2,4 \%)$ respondeu "Não faço ideia". Os desconhecimentos sobre os usos de programação na educação escolar devem ser considerados nas proposições de futuros projetos na instituição.

\section{Conclusão e trabalhos futuros}

Nos últimos anos algumas iniciativas de uso de programação de computadores (e do pensamento computacional) vêm sendo desenvolvidas na educação escolar no Brasil, o que pode ser verificado no levantamento bibliográfico deste artigo. Mas, nos estudos levantados se verifica que pouco se pesquisou sobre as apropriações que os professores de disciplinas propedêuticas estão fazendo em relação ao uso dos recursos de programação como apoio ao aprendizado. Especificamente no ensino técnico as pesquisas são escassas, segundo Silva et. al. (2015) apenas 1\% dos artigos das fontes pesquisadas pelos autores se destinava a essa modalidade de ensino. Daí a importância da presente pesquisa como forma de contribuir para o embasamento das ações dos docentes que atuam nestes cursos.

A investigação ajudou na identificação sobre os conhecimentos dos professores do IFRJ das possíveis ferramentas que podem ser usadas para o desenvolvimento do pensamento computacional e sobre os possíveis benefícios que podem decorrer das ações com os alunos. Os resultados indicam que a maioria dos docentes possuía entendimento das presumidas contribuições que o ensino de programação pode propiciar para o aprendizado dos estudantes do Ensino Médio. Contudo, iniciativas dos docentes para o uso de programação em disciplinas propedêuticas eram poucas, provavelmente pelo constatado desconhecimento de novos recursos de programação que podem ser usados para auxiliar no aprendizado.

Como a maioria dos pesquisados afirmou acreditar em melhorias de aprendizado dos conteúdos que o trabalho interdisciplinar com o uso da programação poderia provocar em relação às disciplinas que lecionam no IFRJ. Isso enseja iniciativas de projetos/oficinas que podem ser propostas, o que para gerar estudos de casos sobre o uso 
V Congresso Brasileiro de Informática na Educação (CBIE 2016)

Anais do XXII Workshop de Informática na Escola (WIE 2016)

de conceitos da Computação nos campi do IFRJ. Dessa forma, a instituição pode se tornar em um difusor de resultados de pesquisas aplicadas sobre conceitos de Computação em disciplinas propedêuticas, desempenhando parte do seu papel institucional.

Este trabalho pode se tornar base para futuras iniciativas de utilização dos recursos de programação, de modo interdisciplinar dentro do IFRJ em turmas de cursos técnicos integrado ao Ensino Médio. E também, para iniciativas de cursos de extensão em parceria com outras escolas de Ensino Médio.

\section{Referências}

Alves, R. M., Sampaio, F. F., Elia, M. F. (2015) "DuinoBlocks: Desenho e Implementação de um Ambiente de Programação Visual para Robótica Educacional", Revista Brasileira de Informática na Educação, v. 22, n. 03, p. 126-140.

Brasil (2008) "Lei no 11.892, de 29 de dezembro de 2008. Institui a Rede Federal de Educação Profissional, Científica e Tecnológica, cria os Institutos Federais de Educação, Ciência e Tecnologia, e dá outras providências.”, Brasília, 2008.

Ferreira, A. C., Melhor, A., Barreto, J., Paiva, L. F., Matos, E. (2015) "Experiência Prática Interdisciplinar do Raciocínio Computacional em Atividades de Computação Desplugada na Educação Básica.", In: Anais do XXI Workshop de Informática na Escola (WIE 2015), p. 256-265.

França, R. S., Amaral, H. J. C. (2013) "Proposta Metodológica de Ensino e Avaliação para o Desenvolvimento do Pensamento Computacional com o Uso do Scratch", In: Anais do XIX Workshop de Informática na Escola (WIE 2013), p. 179-188.

Hinterholz, L. T. , Cruz, M. E. K. (2015) "Desenvolvimento do Pensamento Computacional: um relato de atividade junto ao Ensino Médio, através do Estágio Supervisionado em Computação III", In: Anais do XXI Workshop de Informática na Escola (WIE 2015), p. 137146.

Lessa, V., Forigo, F., Teixeira, A., Licks, G. P. (2015) "Programação de Computadores e Robótica Educativa na Escola: tendências evidenciadas nas produções do Workshop de Informática na Escola", In: Anais do XXI Workshop de Informática na Escola (WIE 2015), p. 93-101.

Rodriguez, C., Zem-Lopes, A. M.; Marques, L.; Isotani, S. (2015) "Pensamento Computacional: transformando ideias em jogos digitais usando o Scratch", In: Anais do XXI Workshop De Informática Na Escola, v. 21, n. 1, p. 62-71.

Scaico, P. D., Lima, A. A., Silva, J. B. B., Azevedo, S., Paiva, L. F., Raposo, E. H., Alencar, Y., Mendes, J. P., Scaico, A. (2013) "Ensino de Programação no Ensino Médio: Uma Abordagem Orientada ao Design com a linguagem Scratch", Revista Brasileira de Informática na Educação, v. 21, n. 02, p. 92-103.

Silva, T. R., Medeiros, T. J., Medeiros, H., Lopes, R., Aranha, E. (2015) "Ensino-aprendizagem de programação : uma revisão sistemática da literatura", Revista Brasileira de Informática na Educação (RBIE), v. 23, n. 1, p. 182-196.

Ventura, M.M. (2007) "O estudo de caso como modalidade de pesquisa”, Revista da Sociedade de Cardiologia do Estado do Rio de Janeiro, v. 5, n. 20, p. 383-386.

Wing, J. M (2006) “Computational Thinking”, Communications of the ACM, v. 49. n. 3, p. 3335 . 